Pacific Journal of Mathematics

THE ASYMPTOTIC SOLUTIONS OF AN ORDINARY
DIFFERENTIAL EQUATION IN WHICH THE COEFFICIENT
OF THE PARAMETER IS SINGULAR 


\title{
THE ASYMPTOTIC SOLUTIONS OF AN ORDINARY DIFFERENTIAL EQUATION IN WHICH THE COEFFICIENT OF THE PARAMETER IS SINGULAR
}

\author{
E. D. CASHWELL
}

1. Introduction. In this paper we are concerned with the solutions, for large values of the complex parameter $\lambda$, of the ordinary differential equation,

$$
w^{\prime \prime}(s)-\left[\lambda^{2} \sigma(s)+\tau(\lambda, s)\right] w(s)=0 .
$$

The variable $s$ ranges over a region in the complex plane in which $\sigma(s)$ possesses a factor $\left(s-s_{0}\right)^{-2}$, where $s_{0}$ is some fixed point of the region. The asymptotic representations of the solutions of an equation formally identical with (1), but in which $\sigma(s)$ contains a factor $\left(s-s_{0}\right)^{\nu}, \nu>-2$, have been considered by Langer [3].

If equation (1) is considered over a region of the complex $s$-plane in which $\sigma(s)$ and $\tau(\lambda, s)$ are bounded, with $\sigma(s)$ bounded from zero, then it is possible to find a pair of asymptotic forms made up of elementary functions, each of these forms representing a solution over the entire region. If, however, $\sigma(s)$ becomes zero in the region under consideration, the asymptotic representations are complicated by the appearance of the Stokes' phenomenon. This necessitates abrupt but determinate changes in the asymptotic forms, if only elementary functions are used, as certain boundaries are crossed in the s- and $\lambda$-planes. The asymptotic representations of the solutions of (1) in this case have been considered by Langer [1] among others, and he has shown the Stokes' phenomenon to be quantitatively dependent upon the order of the zero of $\sigma(s)$. In a later paper [3], the theory was extended to include the cases where $\sigma(s)$ contains a factor $\left(s-s_{0}\right)^{\nu}$, $\nu>-2$, and $\tau(\lambda, s)$ has a pole of first or second order at $s_{0}$. He showed that the Stokes' phenomenon is engendered by and depends upon an infinity in either of the two coefficients in (1).

Received December 21, 1950; presented to the American Mathematical Society April 30, 1949. The author wishes to thank Professor R. E. Langer for suggesting this problem and for his help in the preparation of this paper.

Pacific J. Math. 1 (1951),337-352. 
It is proposed to consider in this paper solutions of equation ( 1 ) in a region which contains as the only singularity of $\sigma(s)$ a pole of second order at a point $s_{0}$, and in which $\sigma(s)$ is bounded from zero while $\tau(\lambda, s)$ has a pole of first or second order at $s_{0}$. Among the functions satisfying an equation of this type we may cite the Bessel functions and certain of the confluent hypergeometric functions.

Although the theory developed by Langer is not applicable to the case presently considered, it is nevertheless found that the broad outlines of the general methods used in the papers mentioned still apply. A differential equation is found which possesses all the essential qualities of (1), and which can be solved explicitly. The solutions of this equation are shown to give asymptotic representations of the solutions of the given equation over definable subregions of the domain in which the coefficients in (1) have the properties assumed above.

In order to arrive at the asymptotic solutions of the given equation, it is found necessary to subdivide the region of large values of $\lambda$ into a finite number of subregions. For $\lambda$ in each of these subregions, and for all admitted values of $s$, two independent asymptotic solutions are derived. Although asymptotic forms of similar structure are derivable for all subregions, the solutions which maintain these forms in the different regions are in general different functions.

2. Hypotheses and normal form of the differential equation. The equation (1) is here considered with the parameter $\lambda$ ranging over any region of the complex plane in which $|\lambda|$ is unbounded. The variable $s$ also is complex, and ranges over a bounded, simply connected domain $R_{s}$ containing a point $s_{0}$ at which $\sigma(s)$ has a pole of second order. Then in some neighborhood of $s_{0}, \sigma(s)$ is of the form

$$
\sigma(s)=\frac{\psi(s)}{\left(s-s_{0}\right)^{2}},
$$

where $\psi(s)$ is a single-valued, analytic function bounded from zero. The constants in the product $\lambda^{2} \psi(s)$, which appears in the first coefficient of (1), are adjusted so that $\psi\left(s_{0}\right)=1$. Expanding $\psi(s)$ about the point $s_{0}$, we have

$$
\psi(s)=1+a_{1}\left(s-s_{0}\right)+a_{2}\left(s-s_{0}\right)^{2}+\cdots \cdot
$$

We assume the conditions a), b), and c) which follow in this section to be satisfied collectively by the coefficients of the differential equation, the domain $R_{s}$, and the range of values of the parameter $\lambda$. The first two of these conditions are : 
a) $\quad \psi(s)$ is a single-valued, analytic function bounded from zero.

b) The coefficient $\tau(\lambda, s)$ has the form

$$
\tau(\lambda, s) \equiv \frac{A_{1}}{\left(s-s_{0}\right)^{2}}+\frac{B_{1}}{s-s_{0}}+C_{1}(\lambda, s),
$$

where $A_{1}$ and $B_{1}$ are constants, and $C_{1}(\lambda, s)$ is an analytic function of $s$, uniformly bounded with respect to $\lambda$. (This condition is precisely the same imposed on $\tau(\lambda, s)$ by Langer in [3] .)

The equation (1) can always be put in a more convenient form by simple changes of the dependent and independent variables.

Letting (cf. [3; p. 399])

$$
s-s_{0}=\frac{z^{2}}{4}, \quad w=z^{1 / 2} u,
$$

we obtain the equation (1) in the form

$$
u^{\prime \prime}(z)-\left[\frac{\rho^{2} \phi^{2}(z)+A}{z^{2}}+\chi(\rho, z)\right] u(z)=0,
$$

where

$$
\begin{gathered}
\rho=2 \lambda, \quad A=4 A_{1}+\frac{3}{4}, \\
\chi(\rho, z)=B_{1}+\frac{z^{2}}{4} C_{1}(\lambda, s), \\
\phi^{2}(z)=1+\frac{a_{1}}{4} z^{2}+\frac{a_{2}}{16} z^{4}+\cdots=1+z^{2} \Phi(z) .
\end{gathered}
$$

The equation (3) is called the normal form of (1), and is the one we shall consider in the following discussion. It is to be observed that if the constants $a_{1}$ and $B_{1}$, appearing in the expressions for $\psi(s)$ and $\tau(\lambda, s)$ respectively, vanish, then equation (1) can be put in normal form (3) by simply translating the origin and changing notation.

Since $\psi(s)$ does not vanish in the domain $R_{s}, \phi^{2}(z) \equiv \psi\left(z^{2} / 4+s_{0}\right)$ does not vanish in the corresponding domain $R_{z}$ in the $z$-plane. Consider the domain $R_{s}$ 
lying on a two-sheeted Riemann surface with branch point at $s_{0}$. Then the transformation $s-s_{0}=z^{2} / 4$ is one-to-one between the bounded, simply-connected domain $R_{s}$ and the corresponding domain $R_{z}$. Denoting by $\phi(z)$ the square root of $\phi^{2}(z)$ which takes the value one when $z=0$, we obtain

$$
\phi(z)=1+b_{1} z^{2}+b_{2} z^{4}+\cdots,
$$

or

$$
\phi(z)=1+z^{2} \phi_{1}(z),
$$

where $\phi_{1}(z)$ is an analytic function of $z$ in $R_{z}$. We are now ready to make the third of our hypotheses :

c) The function $z e^{\Phi_{1}(z)}$ is schlicht, where

$$
\Phi_{1}(z)=\int_{0}^{z} \zeta \phi_{1}(\zeta) d \zeta
$$

Since the function $z e^{\Phi_{1}(z)}$ has a nonvanishing derivative at $z=0$, it is schlicht in some neighborhood of this point. The hypothesis c) in effect restricts the $z$-domain under consideration (and hence $R_{s}$ ) to be one in which this property maintains.

3. The "related" differential equation. Throughout the considerations which follow, the quantities $\left(\rho^{2}+1 / 4+A\right)^{1 / 2}$ and $[\phi(z)]^{1 / 2}$ enter frequently. It serves for notational simplification to denote the former of these by $\mu$, that determination of the root being chosen for which $-\pi / 2<\arg \mu \leq \pi / 2$ when $\rho=0$. We determine $[\phi(z)]^{1 / 2}$ by the condition $[\phi(0)]^{1 / 2}=1$.

In the case where equation (1) is considered over a region in which $\sigma(s)$ is bounded from zero, the asymptotic forms of a pair of solutions can be found, the leading terms of which are (cf. [2], p. 550]).

$$
\frac{1}{[\sigma(s)]^{1 / 4}} e^{ \pm \lambda \int[\sigma(t)]^{1 / 2}} d t
$$

This suggests that, in order to find an approximating equation to equation (3), we consider the functions

$$
y(z)=\left[\frac{z}{\phi(z)}\right]^{1 / 2} e^{ \pm \mu\left[\log z+\Phi_{1}(z)\right]},
$$

where, because of the relative complexity of our equation, it is found necessary 
to the following developments to replace the parameter $\rho$ by $\mu$. A direct calculation shows that

$$
y^{\prime \prime}(z)-\frac{\rho^{2} \phi^{2}(z)+A}{z^{2}} y(z)=\omega(z) y(z)
$$

where

$$
\omega(z)=\frac{\phi^{2}(z)}{4 z^{2}}-\frac{1}{4 z^{2}}-\frac{\phi^{\prime}(z)}{2 z \phi(z)}-\frac{\phi^{\prime \prime}(z)}{2 \phi(z)}+\frac{3}{4}\left[\frac{\phi^{\prime}(z)}{\phi(z)}\right]^{2}+A \Phi(z),
$$

the quantity $\Phi(z)$ in the last term being defined by the relation $\phi^{2}(z)=1+$ $z^{2} \Phi(z)$.

The differential equation (6) appears at first glance to have the same form as equation (3). However, since the denominator of each of the first three terms in the expression for $\omega(z)$ vanishes at the origin, it is necessary to consider this coefficient further. Grouping the first two terms and replacing $\phi^{2}(z)$ by its expression immediately above, and substituting in the third term from (4) for $\phi(z)$, we can write (7) in the form

(8) $\omega(z)=\frac{z^{2} \phi(z)}{4 z^{2}}+\frac{z\left(2 \phi_{1}(z)+z \phi_{1}^{\prime}(z)\right)}{2 z \phi(z)}-\frac{1}{2} \frac{\phi^{\prime \prime}(z)}{\phi(z)}+\frac{3}{4}\left[\frac{\phi^{\prime}(z)}{\phi(z)}\right]^{2}+A \Phi(z)$.

Since $\phi(0) \neq 0$, it follows from (8) that if $\omega(0)$ is defined appropriately, then $\omega(z)$ is analytic throughout $R_{z}$.

In virtue of the analyticity of $\omega(z)$ over $R_{z}$, the differential equation (6) possesses all of the essential qualities of (3). Following Langer's terminology, we refer to the equation (6) as the "related" equation. The formulas (5) give explicitly a pair of independent solutions of this equation.

4. Solutions of the related equation. For convenience, let us define $\xi$ by the formula

$$
\xi=\mu\left[\log z+\Phi_{1}(z)\right] .
$$

With this, the functions (5) which solve the related equation (6) may be written

$$
y_{1}(z)=\left[\frac{z}{\phi(z)}\right]^{1 / 2} e^{\xi}, \quad y_{2}(z)=\left[\frac{z}{\phi(z)}\right]^{1 / 2} e^{-\xi}
$$

The related equation (6) has a regular singular point at $z=0$, with exponents 
$1 / 2 \pm \mu$. For a fixed value of the parameter $\mu$, it is seen that, in a neighborhood of the origin, the formulas (10) are of the form

$$
y_{1}(z)=z^{1 / 2+\mu} O(1), \quad y_{2}(z)=z^{1 / 2-\mu} O(1),
$$

where $O(1)$ stands as usual for a bounded function of $z$.

From the formulas (11) it is seen that, if $R(\mu)>0$, then $y_{1}(z)$ approaches zero as $z$ approaches zero. The function $y_{1}(z)$ is in fact singled out as that solution of equation (6) which vanishes at $z=0$ to a higher order than any other. At $z=0, y_{2}(z)$ on the other hand vanishes or becomes infinite according as $R(\mu)$ is less than or greater than $1 / 2$.

If $R(\mu)<0$, the behaviors of $y_{1}(z)$ and $y_{2}(z)$ in this respect are reversed.

5. The transformation $\xi=\mu\left[\log z+\Phi_{1}(z)\right]$. Consider the transformation

$$
\zeta=z e^{\Phi_{1}(z)} \text {. }
$$

Since the function on the right of the equality sign is schlicht by hypothesis, the domain $R_{z}$ is mapped conformally onto a corresponding domain which contains the origin in the $\zeta$-plane.

Further, let $w$ be defined by the relation

$$
w=\log \zeta .
$$

If the $\zeta$-domain is cut along the axis of negative real numbers, it is mapped in a one-to-one manner by the transformation (13) onto a semi-infinite strip of width $2 \pi(-\pi<d(w) \leq \pi)$ parallel to the real axis in the $w$-plane.

Omitting the intermediate transformation (13), we see that the relation

$$
w=\log z+\Phi_{1}(z)
$$

may be applied directly to the domain $R_{z}$. In order that (14) be a one-to-one transformation, the choice above of the strip in the $w$-plane imposes upon $R_{z}$ a cut, the image of the upper edge of the strip, from $z=0$ to a point on the boundary.

Let $r_{w}$ denote the following subregion of the region in the $w$-plane: the semiinfinite, rectangular strip bounded on the right by the line $R(w)=K$, subject of course to the restriction that the right boundary of $r_{w}$ lie in the fundamental region in the $w$-plane. The image in the $z$-plane of $r_{w}$ is denoted by $r_{z}$.

The transformation (9) maps the region $r_{w}$ conformally onto a region $r_{\xi}$ in the $\xi$-plane. It is evident that the region $r_{\xi}$ is obtained from $r_{w}$ by a magnification with the factor $|\mu|$ coupled with a rotation about the origin through an angle 
$\arg \mu$.

6. Gamma curves. In the region $r_{w}$, denote the lower right corner by $w_{1}^{*}$ and the upper right corner by $w_{2}^{*}$. In order to avoid unnecessary duplications, let us for the moment denote either of these points by $w_{j}^{*}$. Through every point $\mathbb{W}$ of $r_{w}$ there passes a broken line consisting of that part of the horizontal line, $d(w)=$ $d(\mathbb{W})$, contained in $r_{w}$, together with that portion of the bounding segment, $R(w)=K$, connecting this line to the point $w_{j}^{*}$. The images in $r_{z}$ of this set of curves in $r_{w}$ are referred to as the $\Gamma$-curves corresponding to $w_{j}^{*}$. Thus two sets of curves, corresponding to the two values of $j(j=1,2)$, are defined in $r_{z}$.

In $r_{z}$, the $\Gamma$-curves of either set are uniformly bounded in length. For by direct calculation we have

$$
d z=\frac{z}{\phi(z)} d w
$$

From (14) it follows that

$$
|z|=\left|e^{w-\Phi_{1}(z)}\right|
$$

and hence that

$$
|d z| \leq M \cdot\left|e^{w}\right| \cdot|d w|
$$

where $M$ is the least upper bound of

in $R_{z}$.

$$
\left|\frac{1}{\phi(z)} e^{-\Phi_{1}(z)}\right|
$$

As the variable point $w$ traces out a horizontal line in $r_{w}, d(w)$ is constant, and with $\eta=R(w)$ we have

$$
|d z| \leq M e^{\eta}|d \eta|
$$

Also, along the portion of the line $R(w)=K$ bounding $r_{w}$ on the right, let $d(w)=$ $\kappa$. Then we have

$$
|d z| \leq M e^{K}|d \kappa|
$$

From the way in which the $\Gamma$-curves were defined, it follows that, if $\Gamma$ denotes 
any one of these curves of either set, then

$$
\int_{\Gamma}|d z| \leq M \int_{-\infty}^{K} e^{\eta} d \eta+M e^{K} \int_{-\pi}^{\pi} d k=M e^{K}(1+2 \pi) .
$$

Since the term on the extreme right is independent of the particular $\Gamma$-curve chosen, the $\Gamma$-curves are uniformly bounded in length.

7. Solutions of the original equation. We have exhibited the related equation (5) which possesses all of the essential features of the equation (3), and which admits the independent solutions $y_{1}(z), y_{2}(z)$ given by (10). This, as we now proceed to show, enables us to write two formal solutions of (3). The latter equation can obviously be written in the form

$$
u^{\prime \prime}(z)-\left[\frac{\rho^{2} \phi^{2}(z)+A}{z^{2}}+\omega(z)\right] u(z)=\delta(\rho, z) u(z),
$$

where

$$
\delta(\rho, z) \equiv \chi(\rho, z)-\omega(z),
$$

a function bounded uniformly with respect to $\rho$ and analytic in $z$ over the region $r_{z}$. Regarding (15) as an inhomogeneous differential equation, we see that the reduced equation coincides with (6). Thus, using a standard procedure in differential equations, we can describe a pair of independent solutions of (15) by the relations

$$
\begin{array}{r}
u_{j}(z)=y_{j}(z)-\frac{1}{W} \int_{z_{0}}^{z}\left[y_{1}(z) y_{2}\left(z_{1}\right)-y_{2}(z) y_{1}\left(z_{1}\right)\right] \delta\left(\rho, z_{1}\right) u_{j}\left(z_{1}\right) d z_{1} \\
(j=1,2) .
\end{array}
$$

Here $W$ is the Wronskian of $y_{1}(z)$ and $y_{2}(z)$, direct calculation yielding $W=-2 \mu$, while $z_{0}$ is any fixed point in $r_{z}$. To each solution of the equation (6), (17) relates a solution of the equation (3).

With the definitions ${ }^{1}$

$$
Y_{j}(z)=z^{-1 / 2} e^{\mp \xi} y_{j}(z), \quad U_{j}(z)=z^{-1 / 2} e^{\mp \xi} u_{j}(z),
$$

1 It is convenient to use the double sign to indicate the combination of two formulas into one. The upper sign is to be associated with $j=1$, and the lower sign with $j=2$. 
and with $C$ denoting the path of integration in $r_{z}$, the equation (17) takes the form

$$
U_{j}(z)=Y_{j}(z)+\frac{1}{2 \mu} \int_{C} K_{j}\left(\rho, z, z_{1}\right) U_{j}\left(z_{1}\right) d z_{1},
$$

where the kernel of this integral equation, denoted here by $K_{j}\left(\rho, z, z_{1}\right)$, has the following definition:

$$
\begin{aligned}
& K_{j}\left(\rho, z, z_{1}\right) \\
& \quad= \pm z_{1} \delta\left(\rho, z_{1}\right)\left[Y_{j}(z) Y_{3-j}\left(z_{1}\right)-Y_{3-j}(z) Y_{j}\left(z_{1}\right) e^{\mp 2\left(\xi-\xi_{1}\right)}\right] ;
\end{aligned}
$$

$\xi_{1}$ is defined as the image of $z_{1}$ under the transformation (9).

Carrying out the process of iteration on (19), we arrive at the formal expression

$$
U_{j}(z)=Y_{j}(z)+\sum_{n=1}^{\infty} Y_{j}^{(n)}(z),
$$

with

$$
\begin{aligned}
Y_{j}^{(n+1)}(z) & =\frac{1}{2 \mu} \int_{C} K_{j}\left(\rho, z, z_{1}\right) Y_{j}^{(n)}\left(z_{1}\right) d z_{1}, \\
Y_{j}^{(0)}(z) & =Y_{j}(z) .
\end{aligned}
$$

We shall now show that for arg $\mu$ in a suitably restricted range, it is possible to choose $z_{0}$ for $j=1,2$ so that when $|\mu|$ is sufficiently large, the series (21) converges uniformly and hence represents an actual solution of equation (3). In accordance with this, the $\mu$-plane will be subdivided into its four quadrants, and the asymptotic forms of the solutions derived in each quadrant. This particular choice of the subdivision of the $\mu$-plane is in part due to the configuration of $r_{z}$, and in part due to the reversal of the behaviors of $y_{1}(z)$ and $y_{2}(z)$ as the imaginary axis in the $\mu$-plane is crossed.

Case $1,0 \leq \arg \mu<\pi / 2$. First Solution. In (17) let us choose as the path of integration a curve belonging to the set of $\Gamma$-curves corresponding to $w_{1}^{*}$, with $z_{0}=0$. It is to be noted that upon any curve of this set, the quantity $R(\xi)$ increases monotonically with the arc length.

Referring to the equations (10), we observe that

$$
\left|Y_{j}(z)\right|<M \text {, }
$$


where $M$ is a suitable large constant. This results from the fact that $\phi(z)$ is analytic in $r_{z}$ and bounded from zero.

Consider the relation

$$
\left|Y_{1}^{(n)}(z)\right|<\frac{M^{n+1}}{|2 \mu|^{n}} \quad(n=0,1,2, \cdots) .
$$

This, in view of (23), is evidently satisfied for $n=0$. It can be shown in the following manner that the validity of this relation for any $n$ implies it for $n+1$, so that by induction the relation is established for all $n$.

According to (22), with $\Gamma$ denoting the $\Gamma$-curve which forms the path of integration, we have

$$
\left|Y_{1}^{(n+1)}(z)\right|<\frac{M^{n+1}}{|2 \mu|^{n+1}} \int_{\Gamma}\left|K_{1}\left(\rho, z, z_{1}\right)\right| \cdot\left|d z_{1}\right| .
$$

Now let us consider the kernel $K_{1}\left(\rho, z, z_{1}\right)$, which is defined by the formula (20). From (16), the function $\delta(\rho, z)$ is analytic over $r_{z}$ and hence bounded. The relations (23) guarantee the boundedness of $Y_{1}$ and $Y_{2}$. Furthermore, since $R\left(\xi-\xi_{1}\right) \geq 0$ on the path of integration, the exponential term is bounded. It follows that the integral on the right of (25) is bounded, and we have

$$
\left|Y_{1}{ }^{(n+1)}(z)\right|<\frac{M^{n+2}}{|2 \mu|^{n+1}} N .
$$

In this it is clear that $N$ is independent of $n$. Hence if we choose $M$ at least as large as $N$, then we have

$$
\left|Y_{1}^{(n+1)}(z)\right|<\frac{M^{n+2}}{|2 \mu|^{n+1}} .
$$

This completes the induction.

In virtue of the relations (24), it is clear that the infinite series on the right of equation (21) converges uniformly for values of $\mu$ satisfying the inequality $2|\mu|>U$. Furthermore, from (21) it follows that

$$
U_{1}(z)=Y_{1}(z)+\frac{O(1)}{2 \mu}
$$

for large values of $\mu$. Substituting for $Y_{1}(z)$ and $U_{1}(z)$ from (18), we can write 
this equation in the form

$$
u_{1}(z)=y_{1}(z)+z^{1 / 2} e^{\xi} \frac{O(1)}{2 \mu}
$$

Replacing $y_{1}(z)$ by its expression as given in (10), we have

$$
u_{1}(z)=\left[\frac{z}{\phi(z)}\right]^{1 / 2} e^{\xi}\left[1+\frac{O(1)}{2 \mu}\right]
$$

where $|\mu|$ is sufficiently large.

Case 1, $0 \leq \arg \mu<\pi / 2$. Second Solution. To obtain a second solution of (3) for this range of $\mu$, we choose as the curves of integration in (17) the same set of $\Gamma$-curves used in obtaining the first solution, but we now take $z_{0}=z_{1}^{*}$, the point on the boundary of $r_{z}$ which maps into $w_{1}^{*}$ under the transformation (14). On any one of these $\Gamma$-curves, the quantity $R(\xi)$ is monotone decreasing with respect to the arc length.

Consider the relation

$$
\left|Y_{2}^{(n)}(z)\right|<\frac{M^{n+1}}{|2 \mu|^{n}}
$$

where $H$ is a suitably large constant. According to the equations (23), this relation is satisfied for $n=0$. We proceed to show by induction that it is true for all $n$. Assume the relation to be valid for $n$. From (22), it follows that

$$
\left|Y_{2}^{(n+1)}(z)\right|<\frac{M^{n+1}}{|2 \mu|^{n+1}} \int_{\Gamma}\left|K_{2}\left(\rho, z, z_{1}\right)\right| \cdot\left|d z_{1}\right| .
$$

The kernel $K_{2}\left(\rho, z, z_{1}\right)$ is given by the fornula (20). Arguments entirely similar to those employed in showing the boundedness of $K_{1}\left(\rho, z, z_{1}\right)$ in the relation ( 25$)$ may be used here to establish the boundedness of $K_{2}\left(\rho, z, z_{1}\right)$ in (30). In fact, the only significant difference in this latter kernel is in the exponential term, which is bounded since we have $R\left(\xi-\xi_{1}\right) \leq 0$ along the path of integration. It follows that

$$
\left|Y_{2}^{(n+1)}(z)\right|<\frac{M^{n+1}}{|2 \mu|^{n+1}} N
$$

where $N$ is a constant independent of $n$. By choosing $U$ at least as large as $N$, we 
can write (31) in the form

$$
\left|Y_{2}^{(n+1)}(z)\right|<\frac{M^{n+2}}{|2 \mu|^{n+1}} .
$$

The induction is complete.

As in the previous solution, the infinite series appearing on the right of (21) converges uniformly for sufficiently large values of $|\mu|$. This enables us to rewrite (21), for such values of $\mu$, in the form

$$
U_{2}(z)=Y_{2}(z)+\frac{O(1)}{2 \mu} .
$$

If $Y_{2}(z)$ and $U_{2}(z)$ are replaced by their equivalent expressions given in (17), we obtain

$$
u_{2}(z)=y_{2}(z)+z^{1 / 2} e^{-\xi} \frac{O(1)}{2 \mu} .
$$

Substituting from (10) for $y_{2}(z)$, we can write this equation as follows :

$$
u_{2}(z)=\left[\frac{z}{\phi(z)}\right]^{1 / 2} e^{-\xi}\left[1+\frac{O(1)}{2 \mu}\right]
$$

for $|\mu|$ sufficiently large.

The equation (3), as was pointed out for the related differential equation, has a regular singular point at $z=0$, with exponents $1 / 2 \pm \mu$. For large values of $\mu$ satisfying the condition $0 \leq \arg \mu<\pi / 2$, the relations (28) and (33) give the asymptotic forms of a pair of independent solutions of (3). It is easily seen from (28) and (33), for a constant value of $\mu$ in this range, that in the neighborhood of the origin we have

$$
\begin{aligned}
& u_{1}(z)=O\left(z^{1 / 2+\mu}\right) \\
& u_{2}(z)=O\left(z^{1 / 2-\mu}\right) .
\end{aligned}
$$

Since $R(\mu)>0, u_{1}(z)$ is determined uniquely as that solution of the equation (3) which vanishes at $z=0$ to a higher order than any other. The solution $u_{2}(z)$ either vanishes or becomes infinite at $z=0$, according as $R(\mu)$ is less than or greater than $1 / 2$. It is evident that this behavior of $u_{2}(z)$ is assumed by any solution independent of $u_{1}(z)$.

Case $2, \pi / 2 \leq \arg \mu<\pi$. First Solution. For this range of arg $\mu$, let us 
choose as the curves of integration in (17) the $\Gamma$-curves corresponding to $w_{2}^{*}$, with $z_{0}=z_{2}^{*}$, the point on the boundary of $r_{z}$ which is the image of $w_{2}^{*}$ under the transformation (14). Upon any one of these curves, the quantity $R(\xi)$ increases monotonically with the arc length.

Carrying out an induction argument exactly like that used in obtaining the first solution of Case 1, we can establish the relation

$$
\left|Y_{1}^{(n)}(z)\right|<\frac{M^{n+1}}{|2 \mu|^{n}}
$$

for all nonnegative integral values of $n$. here $M$ is a suitably determined constant. The uniform convergence, for sufficiently large values of $\mu$, of the series on the right of (21) follows immediately, yielding the formula

$$
U_{1}(z)=Y_{1}(z)+\frac{O(1)}{2 \mu} .
$$

Just as in the previous case, this can be rewritten in the form

$$
u_{1}(z)=\left[\frac{z}{\phi(z)}\right]^{1 / 2} e^{\xi}\left[1+\frac{O(1)}{2 \mu}\right],
$$

for $|\mu|$ sufficiently large.

Case $2, \pi / 2 \leq$ arg $\mu<\pi$. Second Solution. In order to find the asymptotic form of a solution independent of $u_{1}(z)$, we choose as the curves of integration in (17) the $\Gamma$-curves corresnonding to $w^{*}{ }^{*}$, with $z_{0}=0$. Along any one of these curves, $R(\xi)$ is monotone decreasing with resnect to the arc length.

In a manner which is formally identical with the argument used to establish (29), we arrive at the analogous relation

$$
\left|Y_{2}^{(n)}(z)\right|<\frac{M^{n+1}}{|2 \mu|^{n}}
$$

for all values of $n$, where $M$ is a suitably chosen constant.

The formula (21), the right hand side of which converges uniformly for large values of $\mu$ in virtue of the preceding relation, yields the expression

$$
U_{2}(z)=Y_{2}(z)+\frac{O(1)}{2 \mu} .
$$


By making the appropriate substitutions from (18) and (10), we obtain

$$
u_{2}(z)=\left[\frac{z}{\phi(z)}\right]^{1 / 2} e^{-\xi}\left[1+\frac{O(1)}{2 \mu}\right]
$$

for $|\mu|$ sufficiently large.

Since for values of $\mu$ in the second quadrant we have $R(\mu)<0$, the behavior of $u_{1}(z)$ and $u_{2}(z)$ is quite different from the behavior of the solution having the same asymptotic form in the first quadrant of $\mu$ values. In fact, $u_{2}(z)$ is now singled out as the solution of (3) which vanishes at $z=0$ to a higher order than any other, whereas $u_{1}(z)$ either vanishes or becomes infinite according as $R(\mu)$ is greater than or less than $-1 / 2$. It is to be observed that although the asymptotic forms of the two indenendent solutions in the second quadrant are the same as those found in the first quadrant, the solutions themselves are in general different.

Case $3, \pi \leq \arg \mu<3 \pi / 2$. For arg $\mu$ in this range, the curves of integration in the formula (17) are chosen as the $\Gamma$-curves corresponding to $w_{1}^{*}$. To find the asymptotic expression for $u_{1}(z)$ we take $z_{0}=z_{1}^{*}$, whereas to find the asymptotic form of $u_{2}(z)$ we choose $z_{0}=0$. (Initting the calculations, which are by now familiar, we arrive at the forms:

$$
\begin{aligned}
& u_{1}(z)=\left[\frac{z}{\phi(z)}\right]^{1 / 2} e^{\xi}\left[1+\frac{O(1)}{2 \mu}\right], \\
& u_{2}(z)=\left[\frac{z}{\phi(z)}\right]^{1 / 2} e^{-\xi}\left[1+\frac{O(1)}{2 \mu}\right],
\end{aligned}
$$

for $|\mu|$ sufficiently large.

The behaviors of the two independent solutions in this quadrant of the $\mu$-plane are clearly similar to the behaviors of the corresponding solutions described in Case 2. It will be observed from the choice of $z_{0}$ that the solution $u_{2}(z)$ is the same in the second and third quadrants, while $u_{1}(z)$ is in general quite different in these two regions.

Case $4,3 \pi / 2 \leq \arg \mu<2 \pi$. For values of $\mu$ in this quadrant, the $\Gamma$-curves corresponding to $w_{2}^{*}$ are chosen as the curves of integration in the forn:ula ( 17 ). We take $z_{0}=0$ in deriving the expression for $u_{1}(z)$, and $z_{0}=z_{2}^{*}$ in deriving the expression for $u_{2}(z)$. Omitting the calculations, we arrive at the usual asymintotic 
forms

$$
\begin{aligned}
& u_{1}(z)=\left[\frac{z}{\phi(z)}\right]^{1 / 2} e^{\xi}\left[1+\frac{O(1)}{2 \mu}\right], \\
& u_{2}(z)=\left[\frac{z}{\phi(z)}\right]^{1 / 2} e^{-\xi}\left[1+\frac{O(1)}{2 \mu}\right],
\end{aligned}
$$

for $|\mu|$ sufficiently large.

The pair of solutions in the fourth quadrant of the $\mu-p$ lane described by these forms have the same characteristics as the corresponding pair found in Case 1, and hence we omit the discussion of their behavior. It is to be noted in comparing Cases 1 and 4 that the solution $u_{1}(z)$ is the same, whereas $u_{2}(z)$ in general is different in the two quadrants considered.

We may now summarize the results of this investigation as follows:

THEOREM. For values of $\mu=\left[\rho^{2}+1 / 4+A\right]^{1 / 2}$ in a given quadrant of the complex plane, $(j-1) \pi / 2 \leq \arg \mu<j \pi / 2, j=1,2,3,4$, and for all $z$ in $r_{z}$, the differential equation (3) admits of a pair of solutions $u_{j}(z), j=1,2$, having the forms

$$
\begin{aligned}
& u_{1}(z)=\left[\frac{z}{\phi(z)}\right]^{1 / 2} e^{\xi}\left[1+\frac{O(1)}{2 \mu}\right], \\
& u_{2}(z)=\left[\frac{z}{\phi(z)}\right]^{1 / 2} e^{-\xi}\left[1+\frac{O(1)}{2 \mu}\right], \quad \xi=\mu\left[\log z+\Phi_{1}(z)\right],
\end{aligned}
$$

for values of $|\mu|$ sufficiently large.

The solution with the exponent $1 / 2+\mu$ relative to the origin, denoted above by $u_{1}(z)$, is the same in the first and fourth quadrants of admissable $\mu$ values. The solution, designated by $u_{2}(z)$, with the exponent $l / 2-\mu$ relative to the origin is the same in the second and third quadrants of the $\mu$-plane. In each of these cases, the second solution is in general different in the two regions mentioned. 


\section{REFERENCES}

1. R. E. Langer, On the asymptotic solutions of differential equations, with an application to the Bessel functions of large complex order, Trans. Amer. Math. Soc. 34 (1932), $447-480$.

2. the second order, with special reference to the Stokes' phenomenon, Bull. Amer. Math. Soc. 40 (1934), 545-592.

3. - On the asymptotic solutions of ordinary differential equations, with reference to the Stokes' phenomenon about a singular point, Trans. Amer. Math. Soc. 37 (1935), 397-416.

Ohio State University 


\title{
PACIFIC JOURNAL OF MATHEMATICS
}

\section{EDITORS}

\author{
HERBERT BUSEMANN \\ University of Southern California \\ R. M. RoBINSON \\ Los Angeles 7, California \\ University of California \\ Berkeley 4, California \\ E. F. BECKENBACH, Managing Editor \\ University of California \\ Los Angeles 24, California
}

\section{ASSOCIATE EDITORS}

R. P. DILWORTH
HERBERT FEDERER
MARSHALL HALL

\author{
P. R. HALMOS \\ HEINZ HOPF \\ R. D. JAMES
}

\author{
B $\emptyset$ RGE JESSEN \\ PAUL LÉVY \\ GEORGE PÓLYA
}

\author{
J. J. STOKER \\ E. G. STRAUS \\ KÔSAKU YOSIDA
}

\section{SPONSORS}

UNIVERSITY OF BRITISH COLUMBIA

CALIFORNIA INSTITUTE OF TECHNOLOGY

UNIVERSITY OF CALIFORNIA, BERKELEY

UNIVERSITY OF CALIFORNIA, DAVIS

UNIVERSITY OF CALIFORNIA, LOS ANGELES

UNIVERSITY OF CALIFORNIA, SANTA BARBARA

OREGON STATE COLLEGE

UNIVERSITY OF OREGON

\author{
UNIVERSITY OF SOUTHERN CALIFORNIA \\ STANFORD UNIVERSITY \\ WASHINGTON STATE COLLEGE \\ UNIVERSITY OF WASHINGTON

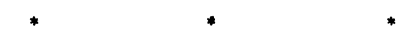 \\ AMERICAN MATHEMATICAL SOCIETY \\ NATIONAL BUREAU OF STANDARDS, \\ INSTITUTE FOR NUMERICAL ANALYSIS
}

Mathematical papers intended for publication in the Pacific Journal of Mathematics should be typewritten (double spaced), and the author should keep a complete copy. Manuscripts may be sent to any of the editors. All other communications to the editors should be addressed to the managing editor, E. F. Beckenbach, at the address given above.

Authors are entitled to receive 100 free reprints of their published papers and may obtain additional copies at cost.

The Pacific Journal of Mathematics is published quarterly, in March, June, September, and December. The price per volume (4 numbers) is $\$ 8.00$; single issues, $\$ 2.50$. Spécial price to individual faculty members of supporting institutions and to members of the American Mathematical Society: $\$ 4.00$ per volume; single issues, $\$ 1.25$.

Subscriptions, orders for back numbers, and changes of address should be sent to the publishers, University of California Press, Berkeley 4, California.

UNIVERSTTY OF CALIFORNIA PRESS • BERKELEY AND LOS ANGELES 


\section{Pacific Journal of Mathematics}

\section{Vol. 1, No. $3 \quad$ BadMonth, 1951}

R. P. Boas, Completeness of sets of translated cosines ............. 321

J. L. Brenner, Matrices of quaternions . ..................... 329

Edmond Darrell Cashwell, The asymptotic solutions of an ordinary differential equation in which the coefficient of the parameter is

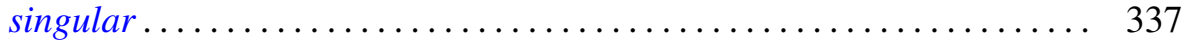

James Dugundji, An extension of Tietze's theorem ................ 353

John G. Herriot, The polarization of a lens ...................... 369

J. D. Hill, The Borel property of summability methods ............... 399

G. G. Lorentz, On the theory of spaces $\Lambda \ldots \ldots \ldots \ldots \ldots \ldots \ldots \ldots \ldots . \ldots \ldots$

J. H. Roberts and W. R. Mann, On a certain nonlinear integral equation of the Volterra type ................................. 431

W. R. Utz, A note on unrestricted regular transformations . . .......... 447

Stanley Simon Walters, Remarks on the space $H^{p} \ldots \ldots \ldots \ldots \ldots \ldots . . \ldots 55$

Hsien Chung Wang, Two theorems on metric spaces ............... 473 